\title{
TREND ANALYSIS OF GLOBAL STOCK MARKET LINKAGE BASED ON A DYNAMIC CONDITIONAL CORRELATION NETWORK
}

\author{
Kedong YIN ${ }^{1}$, Zhe LIU $^{2}$, Peide LIU $^{3}$ \\ ${ }^{1,2}$ School of Economics, Ocean University of China, \\ Songling Road 238, 266100 Qingdao Shandong, China \\ ${ }^{3}$ School of Management Science and Engineering, Shandong University of Finance \\ and Economics, Erhuandong Road 7366, 250014 Jinan Shandong, China \\ E-mails: ${ }^{1}$ yinkedong@ouc.edu.cn; ${ }^{2}$ lwsge@126.com; \\ 3peide.liu@gmail.com (corresponding author)
}

Received 03 March 2017; accepted 09 June 2017

\begin{abstract}
The paper analyses the trend of global stock market linkages via daily data of 51 stock indices spanning the period 22 July 2005 to 30 June 2016 which covers four regions: America, Europe, Asia Pacific and Africa. A dynamic conditional multivariate generalized autoregressive conditional heteroskedasticity (DCC-MVGARCH) approach was used to calculate dynamic correlation coefficient in order to construct the volatility networks. The methods of minimum spanning tree (MST) and low pass filter were for the first time applied to analyze the variable periodicity of the comovement. The original contribution of this paper is that contrary to previous works, financial events such as Quantitative Easing (QE) and Bailouts are accounted for rather than only crisis factors such as the 2008 financial crisis and the European Debt crisis. The main findings of the paper are as follows: (1) Financial crisis promotes and strengthens global stock markets linkage in the short run; (2) Linkage cycles post crisis are significantly short, due to the effect of monetary policy spillover effects caused by QE from developed to developing countries; and (3) European stock markets are the information transmission hub for global stock market. The research conclusions would be significant for both government to regulate markets as well as for investors to diversify risks.
\end{abstract}

Keywords: complex network, DCC-MVGARCH, topological properties, minimum spanning tree, low pass filter, variable periodicity analysis.

JEL Classification: C15, C61, E32, G01, G14, G15.

\section{Introduction}

In the context of financial globalization, the development and policy formulation of a country's financial market will have a certain impact on the financial markets of other countries. Global stock market linkage refers to the common movement of stock markets. Prices in different stock markets will eventually achieve long-term equilibrium with different market yields and price fluctuations have a strong correlation. It is significant for both securities investors to diversify risks as well as for government to 
regulation market by making an intensive study of the dynamic changes of the relationship between international stock markets.

Complex networks theory is useful in the complexity and non-linear relationships study of a system (Fan 2014). Network science has its origin in graph theory, topology and other branches of applied mathematics. In the middle of the $20^{\text {th }}$ century, Erdös-Rény (ER) random graph theory, the basic model of stochastic networks, became the most powerful tool for studying real networks. With the proposal of the small-world and scale-free network models, the traditional ER theoretical framework has been transcended and real-world statistics has been characterized more effectively (Fang et al. 2007). Subsequently, network science has been applied in many fields, such as sociology (Porat, Benguigui 2016), biology and medicine (Wang et al. 2016; Huang et al. 2017), physics (Sui et al. 2016), information dissemination (Carley et al. 2016; Park et al. 2016), interdisciplinary knowledge exchange (Shan et al. 2014), technology integration (Lee et al. 2014), and art design (Jiang 2016). Almost at the same time, the application of complex network analysis were introduced into financial markets (Mantegna 1999) and has been widely applied to many fields as macroeconomic indices (Gao et al. 2013), business cycles (Caraiani 2013), stock markets (Hwang et al. 2016; Brida et al. 2016; Coletti 2016; Majapa, Gossel 2016; Qiao et al. 2016), foreign exchange (Naylor et al. 2007; Brida, Risso 2010) and financial market risk (Huang et al. 2016).

The univariate GARCH model is widely used in the field of market volatility and risk description due to the heterogeneity of financial time series. However, the univariate GARCH model cannot satisfy the research on different market or assets with distinguishing influencing factors. Then, the multivariate GARCH models, as a tool which can both reflect the fluctuation characteristics of univariate GARCH model and describe the interaction and correlation between different variables and different factors, were introduced into the financial market analysis. Dynamic conditional correlation multivariate generalized autoregressive conditional heteroskedasticity (DCC-MVGARCH) (Engle 2002) is the improved model of CCC-MVGARCH (Bollerslev 1990) and BEKK-MVGARCH (Engle, Kroner 1995). Compared to other GARCH models, DCC-MVGARCH is intuitive, concise and stable. The biggest characteristic of the DCC model is that the correlation coefficient is the dynamic correlation coefficient which changes over time. There are many scholars who apply the model to analyze the dynamic relationship between economic indicators and financial assets to explore the internal mechanism of economic phenomena (Chandra 2005; Aslanidis et al. 2010).

In this paper, we use the 51 most representative stock indices to analyze dynamic changes in global stock market linkage. After transforming the 51 indices' closing price into log return, the dynamic coefficient correlations are calculated through DCC-MVGARCH model. Then, we construct the volatility network and analyze their topological features, such as the network density, average path length and clustering coefficient. Further, by the algorithm of minimum spanning tree and low pass filter, we analyze the variable periodicity of the comovement. Finally, using the index of average weighted degree, we investigate the stability of the network and confirm the cluster structures of global stock markets with core nodes for every cluster.

The remainder of the paper is organized as follows. Section 1 describes the literature 
on the dynamic analysis of global stock market linkage. Section 2 discusses the data set and the methodology we applied. In Section 3, we present the principal empirical results and analyses. Final section summarizes and concludes the paper.

\section{Literature review}

For theoretical research, the interaction mechanism of global stock market linkage can be summarized as the "economic fundamental hypothesis" and the "market contagion hypothesis". The economic fundamental hypothesis is based on the traditional financial theory that investors are rational and argues that asset price linkage is caused by asset fundamentals (Cho et al. 1986; Contessi et al. 2008). For the market contagion hypothesis, researchers believe that not only macroeconomic factors but also investor decisions contribute to the linkage of stock markets (Connolly, Wang 2003). At the empirical research level, studies of international stock market linkage are primarily on the basis of returns in specific countries or regions through the regression-based econometric methods, such as the multivariate vector autoregressive (VAR) method (Eun, Shim 1989; Neaime 2006; Guyot et al. 2014), multivariate generalized autoregressive conditional heteroskedasticity (MVGARCH) (Égert, Kočenda 2007; Xu et al. 2010; Neaime 2006), co-integration and error correction models (ECMs) (Bessler, Yang 2003; Mylonidis, Kollias 2010; Neaime 2012, 2016), directed acyclic graphs (DAGs) (Liang et al. 2015) and the couple method (Gong, Huang 2009). Although the measurement method in global stock market linkage has been applied extensively and has yielded some meaningful conclusions, there are still some limitations. First, the regression model is limited by the properties of the sequence, such as autocorrelation, nonstationarity and heteroskedasticity, which lead the result of parameter estimation might not be robust. Then, the diversity of parameters and indices in the model might lead to diversification of the results. Further, the regression-based approach primarily examines linear Granger causality, but lacks explanatory power for more complex interactions among actual global stock markets.

With the development of network science, increasing numbers of researchers are attempting to study global stock market linkage from a network perspective. The market commodities are commonly set as nodes and the interrelationships defined as the correlation coefficient of two stocks' logarithmic returns as links. Two kinds of correlation coefficients have been distinguished in previous studies as static and dynamic. Then, the study of financial variables becomes the study of a network's topological properties. Threshold and non-threshold networks are the principal tools for studying a particular correlation network. The first is typically based on the principal features of networks, such as degree distribution, clustering coefficient and average path length (Nobi et al. 2014) as well as degree-degree correlations, betweenness and modularity (Boginski et al. 2005; Huang et al. 2009). The second is based on applying algorithms to the correlation network, such as minimum spanning trees (MSTs) (Jung et al. 2006; Aste et al. 2010; Garas, Argyrakis 2007; Majapa, Gossel 2016), hierarchical trees (HTs) (Tabak et al. 2010; Cheong et al. 2012), planar maximally filtered graphs (PMFGs) (Tumminello et al. 2005), average linkage-based MSTs (Tumminello et al. 2007) and dynamic spanning trees (Sensoy, Tabak 2014). 
However, the aforementioned methods can lead to different results as a result of different settings of correlation coefficients. Many researchers have focused on a rolling correlation process (Buttfield, Bolton 2005) to establish dynamic correlation coefficient networks (Wang, Xie 2015; Ma et al. 2016), but because of the researchers' specific choices regarding parameters such as the length and drift of the estimation window, there have been multifarious results (Karolyi, Stulz 1996; Chan 2006; Neely, Weller 2000). In addition, as stock markets typically exhibit increased volatility, the correlation coefficient estimate can be exposed to contortion and encounter severe upward bias. In this study, we calculate the dynamic correlation coefficient by DCC-MVGARCH model suggested by Engle (2002). On the one hand, in contrast to the rolling correlation process, the model parameters need not be set, which can eliminate the impact of subjective parameter settings (Lyócsa et al. 2012). On the other hand, the consequences calculated by DCC-MVGARCH are based on full samples and the parameter estimation is unbiased, even if there is heteroskedasticity in the data.

To sum up, firstly, comparing with traditional econometric models, the results of global stock market linkage research from a global perspective by complex networks are relatively few. Then, scholars focus on the situation before and after the financial crisis primarily but lack of the detailed study of other financial events, such as Quantitative Easing (QE) and Bailouts. In addition, there have been a massive research focus on network analysis without economic analysis. In this paper, we fuse DCC-MVGARCH volatility network, minimum spanning tree and low pass filter for the first time to do the trend analysis of global stock market linkage. Through the combination of econometric model and network, the limitation of single method is overcome. The financial events such as Quantitative Easing (QE) and Bailouts are also accounted into the analysis in order to get a more comprehensive conclusion. The evolution of global stock market volatility linkage network topological properties are studied through the threshold networks. Then, by calculating the average distance of minimum spanning tree derived from non-threshold networks, we make an empirical test on the variable periodicity of the global stock market linkage. Finally, we investigate the stability of networks and confirm the core node for every cluster.

\section{Data set and applied methodology}

\subsection{Data set}

Our sample consists of daily closing price observations of 51 specific stock indices which cover four regions: America, Europe, Asia Pacific and Africa for the period July 222005 to June 30 2016. The specific distribution of these indices are shown in Table 1. All of these data sets are taken from the Wind information database. July 222005 is choose as the starting point owing to the sample of China reforms the exchange rate system on July 21 2005. As different zones have different holidays and the trading days exist difference, we first match the data according to the date and then fill the missing data arising from holidays and special events as the average of the recorded previous price and the next price. 
Table 1. Major elements of global stock indices

\begin{tabular}{ll}
\hline \multicolumn{1}{c}{ Areas } & \multicolumn{1}{c}{ Stock Indices } \\
\hline America & ISPA, MERV, SPX, GSPTSE, MXX, IXIC, NYA, IBOVESPA, IBVC \\
\hline Europe & $\begin{array}{l}\text { ISEQ, ATX, BLOM, BFX, BUX, PX, KAK, GDAXI, RTS, FCHI, HEX, AEX, } \\
\text { WIG, LUXXX, OSEAX, BVLX, OMXSPI, SSMI, IBEX, ASE, ITLMS, UKX }\end{array}$ \\
\hline Asia Pacific & $\begin{array}{l}\text { DFM, AORD, KLSE, STI, KS11, HIS, VNINDEX, PSI, SENXEX, N225, } \\
\text { SASEIND, 000001, 399001, SETI, NZ50, JKSE, XU100, TA100 }\end{array}$ \\
\hline Africa & CASE, NGSEINDX \\
\hline
\end{tabular}

\subsection{Applied method}

\subsubsection{DCC-MVGARCH}

We use the DCC-MVGARCH model proposed by Engle (2002) to calculate dynamic conditional correlation coefficients. First, transform the daily closing price as the daily logarithmic return by

$$
R_{\mathrm{i}}(t)=\ln I_{i}(t)-\ln I_{i}(t-1),
$$

where $I_{i}(t)$ is the closing price of index $i$ on day $t$. Then, the DCC-MVGARCH model can be set under the assumption that the innovation sequence of $k$ kinds of indices' return $\left\{e_{t}\right\}$ is an independent and identically distributed white noise process with a zero mean, and the covariance matrix $H_{t}$ obeys a multivariate normal distribution as follows:

$$
\begin{gathered}
r_{t}=u_{t}+e_{t} ; \\
e_{t} \mid \Omega_{t-1} \sim N\left(0, H_{t}\right) ; \\
H_{\mathrm{t}}=D_{t} R_{t} D_{t} ; \\
Q_{t}=\left(1-\sum_{m=1}^{M} \alpha_{m}-\sum_{n=1}^{N} \beta_{n}\right) \bar{Q}+\sum_{m=1}^{M} \alpha_{m}\left(\varepsilon_{t-m} \varepsilon_{t-m}^{\prime}\right)+\sum_{n=1}^{N} \beta_{n} Q_{t-n},
\end{gathered}
$$

where $\Omega_{t-1}$ is the information collection of $r_{t}$ at time $t, \bar{Q}=T^{-1} \sum_{t=1}^{T} \varepsilon_{t} \varepsilon_{t}^{\prime}$ is the unconditional variance matrix of standardized residuals, $R_{t}=\left(Q_{\mathrm{t}}^{*}\right)^{-1} Q_{t}\left(Q_{\mathrm{t}}^{*}\right)^{-1}$, and $Q_{\mathrm{t}}^{*}$ contains the elements on the diagonal of $Q_{t}$, as follows:

$$
Q_{t}^{*}=\left|\begin{array}{cccc}
\sqrt{\mathrm{q}_{11}} & 0 & \cdots & 0 \\
0 & \sqrt{q_{22}} & \cdots & 0 \\
\vdots & \vdots & \ddots & \vdots \\
0 & 0 & \cdots & \sqrt{q_{k k}}
\end{array}\right|,
$$

then, $R_{t}$ is the matrix of the dynamic correlation coefficient, $D_{t}=\operatorname{diag}\left(\sqrt{h_{i t}}\right)$, where $h_{\mathrm{i} i, t}=\omega_{i}+\sum_{p=1}^{P_{i}} \alpha_{i p} e_{i t-p}^{2}+\sum_{q=1}^{Q_{i}} \beta_{i q} h_{i t-q}$, which means that every index's return undergoes a $\operatorname{GARCH}(p, q)$ process. Finally, the correlation coefficient between indices $i$ and $j$ can 
be expressed as

$$
\rho_{i j, t}=\frac{q_{i j, t}}{\sqrt{q_{i i, t} q_{j j, t}}}, i, j=1,2, \cdots, n, i \neq j .
$$

The estimation of the DCC-MVGARCH model can be divided into two steps, estimating the univariate GARCH process for each asset, and then estimating the dynamic correlation coefficient by the standardized residuals obtained from step 1. A test of aerial correlation and heteroskedasticity is required before the estimation of the model. In this paper, we use the R package MTS (Tsay 2014) to perform the DCC-MVGARCH estimation and derive the dynamic correlation coefficient.

\subsubsection{Threshold network}

A threshold network removes the weak correlation by setting a threshold, so that the network is simplified and the structure of the network can be better studied. Previous studies use different thresholds for different financial markets, such as above 0.7 in a price index network analysis (Chi et al. 2010; Liu, Tse 2012) or above 0.3 in a static correlation coefficient stock market network (Nobi et al. 2014). There are few studies of threshold setting for the dynamic correlation coefficient in the current literature.

In order to find the perfect threshold for the network, given a random variable $r, r \in$ $[0,1]$, calculate the number of edges $E_{i}(r)$ and the number of non-isolated vertices $V_{i}(r)$ with reference to the static correlation coefficient network threshold. The delta is calculated as

$$
\begin{gathered}
\alpha_{i}=\frac{E_{i}(r)}{E_{i}(0.3)}-1, i=1,2, \cdots, n ; \\
\beta_{i}=\frac{V_{i}(r)}{V_{i}(0.3)}-1, i=1,2, \cdots, n .
\end{gathered}
$$

As the empirical results, the change of points is always smoothly downward, with the result that the determinant of the threshold is the change rate of the number of vertices. The change of vertices change rate is defined as

$$
\Delta \beta_{i}=\frac{\beta_{i+1}}{\beta_{i}},
$$

and the first obvious jump points is chosen as the threshold.

\subsubsection{Network topological properties}

To conduct the analysis of network topological properties, the distance between vertex and vertex is defined as the Euclidean distance,

$$
d_{i j}=\sqrt{2\left(1-r_{i j}\right)}
$$

where $r_{i j}$ is the correlation coefficient obtained from the DCC-MVGARCH model. This distance formula is also applicable to the following MST method.

The network density is defined as the ratio of the number of links in a network to the maximum number of links if every pair of vertices was to have an edge between them, as follows: 


$$
\rho=\frac{2 M}{N(N-1)},
$$

where $M$ is the number of edges that actual exist in the network, and $N$ is the number of vertices.

The average path length is the shortest distance of all pairs of vertices. The distance here is defined as the sum of edges' weights, where the edges constitute the shortest path from one node to another. The average length path can be expressed as

$$
A P L=\frac{2}{N(N-1)} \sum_{i \neq j \in V} d_{i j},
$$

where $d_{i j}$ is the shortest distance between vertices $i$ and $j$.

The clustering coefficient is a parameter that measures the degree of a group. For a node $i$ in a $k$-group, $L=\left[x_{1 i}, x_{2 i}, \cdots, x_{n i}\right],(n \geq 2)$ is the $n$-element set of vertices that have a connection with node $i$. For these vertices, there are at most $n(n-1) / 2$ connections. The clustering coefficient is the ratio of the number of connections that actually exist in the network of these vertices to the theoretical maximum of connections between these vertices. The formula for computing the clustering coefficient is

$$
C_{i}=\frac{2 E_{i}}{k_{i}\left(k_{i}-1\right)},
$$

where $E_{i}$ is the number of edges that exist in the network between the vertices connecting with node $i$, and $k_{i}$ is the degree of node $i$. If there is a node that has no edge or has only one edge with any of the other nodes, the clustering coefficient is set to zero. For the entire network, the clustering coefficient is defined as the arithmetic mean of all nodes' clustering coefficients, calculated as

where $N$ is the number of nodes.

$$
C=\frac{1}{N} \sum_{i=1}^{N} C_{i},
$$

\subsubsection{Minimum spanning tree}

The MST used here is the minimum weighted spanning tree. The tree $T$ is the MST of a weighted undirected graph $G$, expressed as

$$
\min w(T)=\sum_{(u, v) \in T} w(u, v),
$$

where $G=(V, E)$ is a weighted undirected graph representing the edge connecting vertices $u$ and $v$, where $w(u, v)$ is the weight of edge $(u, v)$. An MST is a minimal connected subgraph of $G$ that has all of the vertices of $G$ with no ring. There are two algorithm as Prim and Kruskal to structure the minimum spanning tree and the Kruskal algorithm is chosen in this paper. 


\section{Principal results and analyses}

We obtain the dynamic correlation coefficient as a $2,855 \times 2,601$-dimensional matrix by DCC-MVGARCH model. The number of rows here means that the time series data contain 2,855 days and for every line, the 2,601 elements can be transformed into a 51×51-dimensional matrix. Then, dynamic correlation coefficient networks can be established with every transformed matrix as the connection matrix. Finally, we obtain 2,855 weighted networks with the value of the correlation coefficient as the weight of the edge and perform the following analysis.

\subsection{Network topological properties analysis}

As mentioned above, to perform a better analysis of the relationship and structure among global stock markets, different from the single network, we try to find the best threshold all of the networks and simplify them. A rough estimate of our approach is to calculate the mean of the change of vertices' change rate for all of the networks with the threshold set of a sequence from 0.01 to 0.99 in steps of 0.01 . Figure 1 shows the relative changes of the vertices for different thresholds. The first jump point is marked “*”, and the value of 0.48 is chosen as the threshold for our networks. Then, for all 2,855 of the networks, if the correlation coefficient between two stock markets' indices is not less than 0.48 , there is an edge between them.

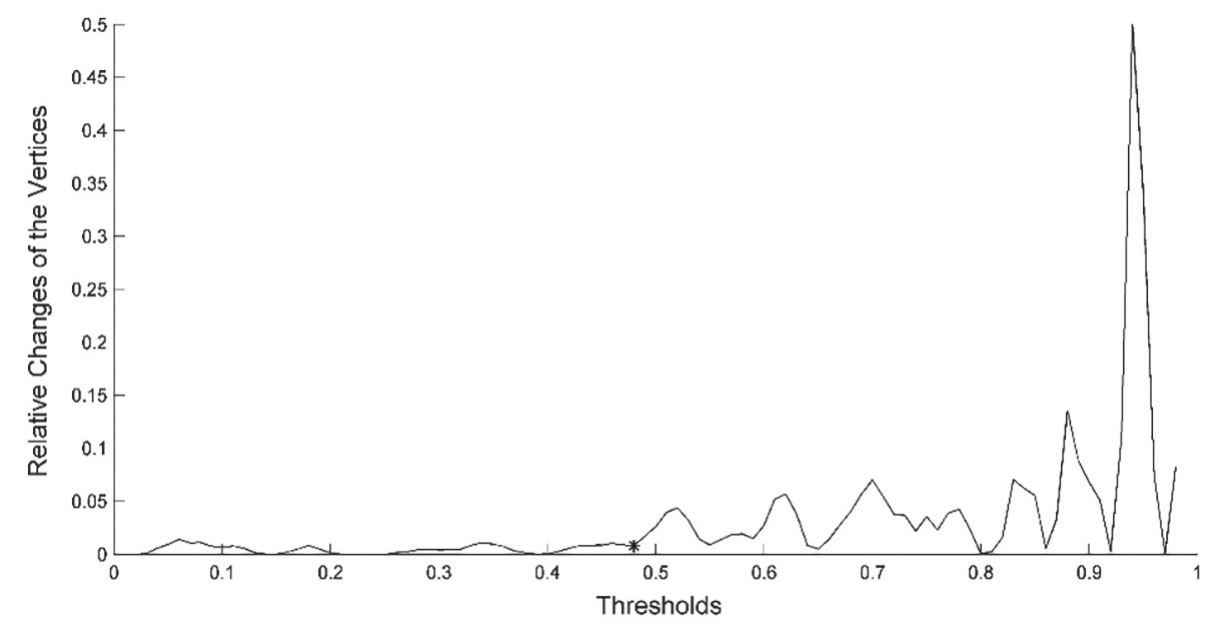

Fig. 1. Relative changes of vertices for different thresholds

\subsubsection{Network density}

Figure 2 shows the network densities of global stock markets. At the beginning, the network density is at the mean level of the fluctuation interval. Then, the variable reflects a fluctuating upward trend until the end of 2008, because of the accelerated process of global integration makes capital flowing freely in international stock markets. When capital is transferred from one stock market to another, the network density rises and then gradually decreases, with the result that the network densities show a regular 


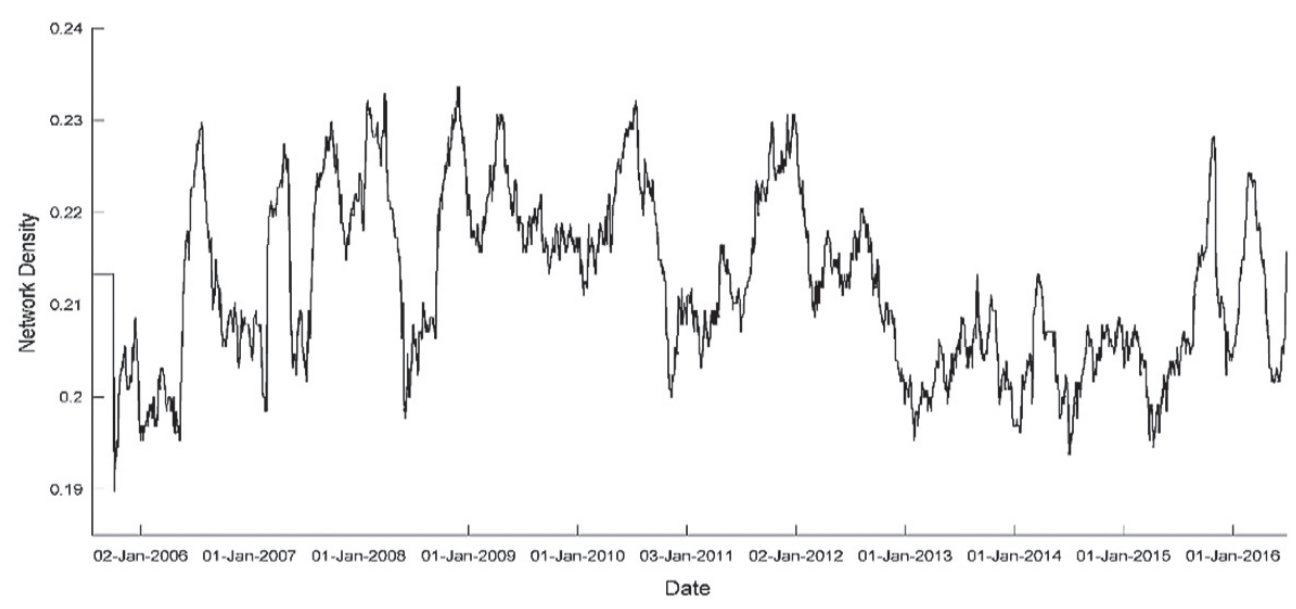

Fig. 2. Network densities of global stock markets

fluctuation. The highest point of the curve appears after the financial crisis, just as in previous studies (Garas, Argyrakis 2007), which is caused by the herd behaviour occurs when financial markets experience a crisis.

Owing to the continuing impact of the financial crisis and the following European debt crisis, the level of the network densities is relatively high from the beginning of 2009 to mid-2012. In this period, the first peak value is at approximately that of mid-2010, reflecting lagging influence and the development of the European debt crisis. The second peak value is at approximately the end of 2011. Differently from crisis factors, the driver here is the European bailout plan. For international capital flows, the crisis and the stimulus plan play the same role, as both of them cause a capital outflow from one market to another.

The time interval from mid-2012 to mid-2015 can be regarded as the third part. During this period, the network densities are at a relatively low level, meaning that the financial markets are recovering from the crisis, and that the global stock markets are in a relatively stable state.

From mid-2015 to the end is the fourth part of the network density series which exhibits a further high and volatile trend. On the one hand, along with the recovery of the world economy, the network densities return to pre-crisis levels. On the other hand, because of the European QE, the capital markets again show large capital flows.

\subsubsection{Average path length}

The global stock markets' average path length at the threshold of 0.48 is shown in Figure 3. As one important measure of one network's transfer efficiency, we calculate it using the Euclid distance instead of the traditional method of the number of edges. From mid-2005 to approximately the end of 2008, the average path length shows a fluctuating downward trend different from the case of network density. It is easy to understand that the transmission efficiency improves because of the upward trend of network density, 


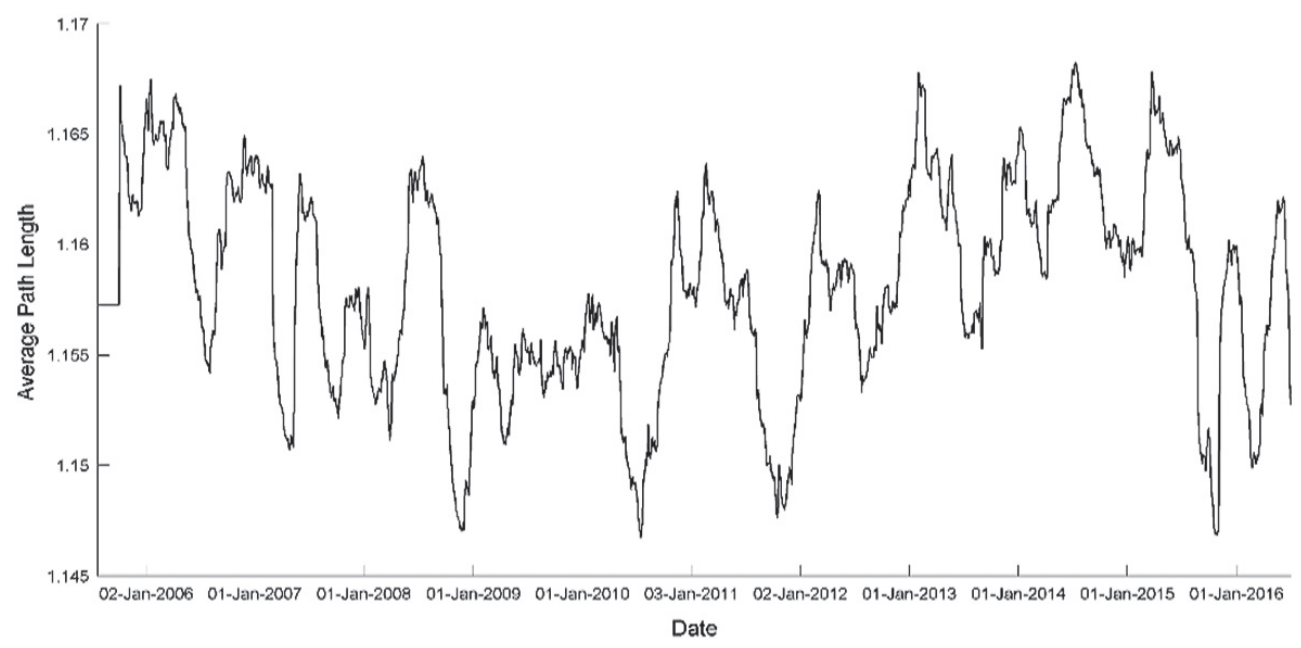

Fig. 3. Average path lengths of global stock markets

as many researchers regard it as a consequence of intensive economic globalization (Eryigit M., Eryigit R. 2009). The lowest point after the financial crisis appears at the end of 2008, indicating the influence of the crisis, with all of the investors becoming extremely sensitive to the information and the spillover effect of different markets being obviously enhanced.

As a result of the financial crisis and the following European debt crisis, the average path length is almost in a continuing low volatility from 2009 to 2011, meaning that information can reach another market very quickly and that there are strong correlations among the stock markets.

Compared with the inconspicuous trend of the network density, the average path length in the third stage from 2012 to mid-2015 is a clear upward trend, meaning that the global stock markets' correlation becomes weak, and the efficiency of information dissemination between different markets decreases.

From mid-2015 to the end of the time series, the average path length shows a process of rapid decline and then slow rising. Then, global stock markets gradually become sparse again.

\subsubsection{Clustering coefficient}

The clustering coefficients of global stock markets shown in Figure 4 do not show a clear evolutionary tendency, and the extent of fluctuation is relatively small. As the clustering coefficient describes the group property of complex networks, the consequence is that the clustering phenomenon does not show a substantial degree of change. The empirical analysis is that regional stock markets typically constitute a group, and when a crisis comes, cross-regional market information spillover can add some of the other areas' stock markets to the major group of global stock markets, thereby causing changes in the clustering coefficient. 


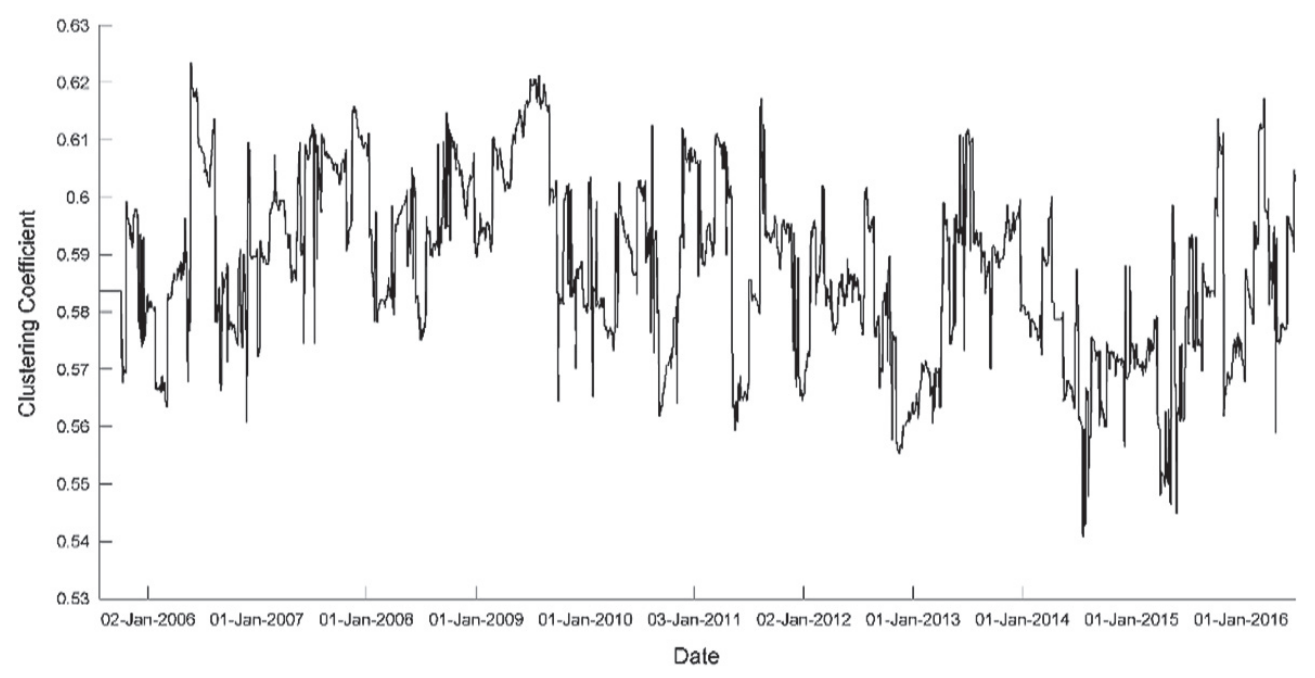

Fig. 4. Clustering coefficients of global stock markets

An interesting concept associated with the clustering coefficient is the "small-world" network, which is determined by the characteristic path length and clustering coefficient. In this paper, we use the average path length as a substitute variable for the characteristic path length combined with the actual situation. Then, the small-world property requires an inverse relationship between the average path length and the clustering coefficient and a small average path length with a large clustering coefficient. Combining the two curves above, it is clear that the networks during the financial crisis and the European debt crisis have the small-world characteristic, meaning that the information transmission speed is very high, and the performance of the network is greatly changed with few changes in the global stock market system in this period.

\subsection{Global stock market linkage estimates}

According to the previous topological studies, there are volatility, tendency, and periodicity in the dynamic evolution of the global stock market network and financial events play a key role. To explore the dynamic changes in the global stock market linkage effects further, we use MSTs to simplify the network structure and then obtain the average of the edges' weights, called the average distance, as the indicator for interpreting dynamic evolution information in a series of networks without a threshold set.

Figure 5 shows the dynamic average distance of global stock markets throughout the entire study period. Here, we refer to the trend line technology in the stock market, marked in the figure by a long dashed line. Differently from previous research, we consider for the financial events not only the crisis factor but also the measures of QEs and other bailout behaviour. All of these events are marked in Figure 5 with the relative number in the time series by the vertical short dashed line and also by the names of these events. It is clear that the dynamic average distances of global stock markets have a volatile nature. Further, low frequency fluctuation plays the dominant role in the wave, and it is 
only on some special occasion or for a short time that the curve shows a high frequency fluctuation, which can be the key factor in the following periodicity analysis.

There are three obvious trends and one possible trend that can determine four stages of the time series. The first stage is defined from the beginning to approximately the $822^{\text {nd }}$ observation, the 2008 financial crisis. In this stage, the linkage of the global stock markets tends to be enhanced as the consequence of economic globalization. One important financial event in this period is the subprime mortgage crisis in the midterm, but that did not change the trend of the curve. This phenomenon can be regarded as the evidence that though the subprime mortgage crisis was the trigger of the financial crisis, the markets did not respond to the crisis sufficiently.

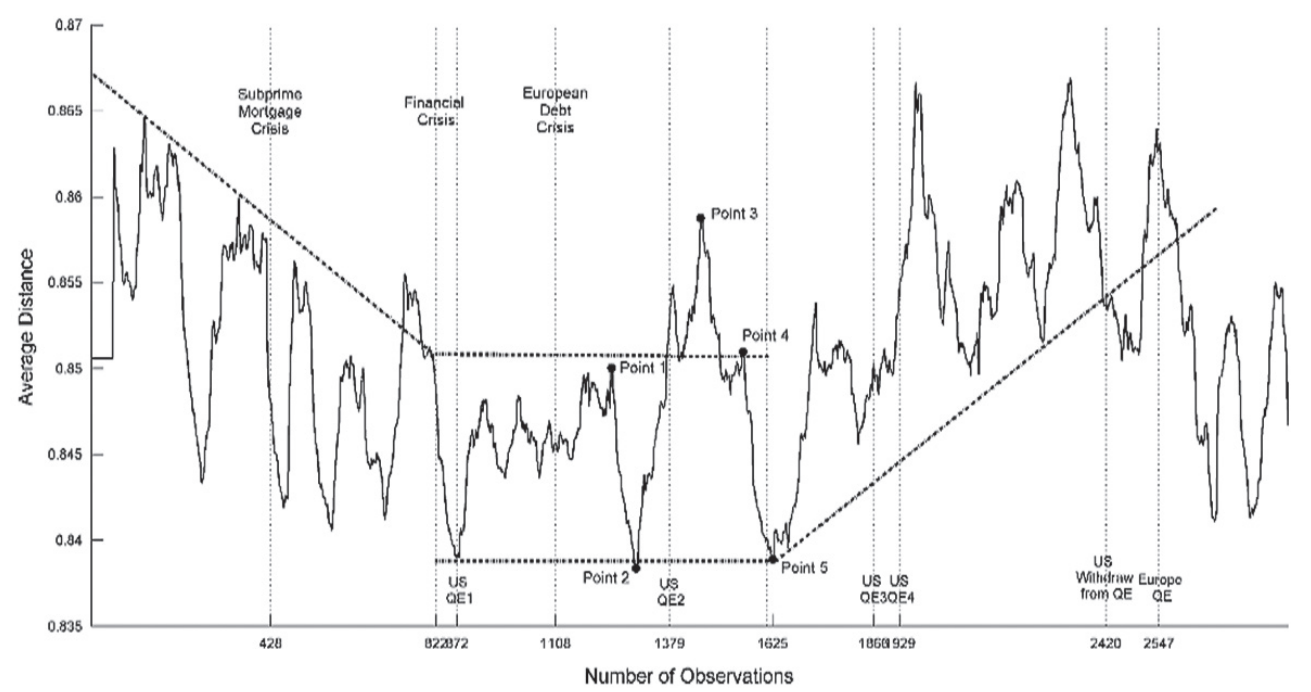

Fig. 5. Average distance of global stock markets

The second stage is from the 2008 financial crisis to the $1,627^{\text {th }}$ observation, and the dynamic linkage trend in this period is interval fluctuation. Because of the financial crisis, the linkage of global stock market rise sharp in a short time until the lowest point of the average distance at the $872^{\text {nd }}$ observation, U.S. QE1. Then, the stock market comovements gradually decrease and form an "M" structure at a relatively high level in the fluctuation range. However, the European debt crisis undermined this trend and weakened the rising energy. After more than one and a half years, the linkage of the stock markets returns to the level before the crisis at Point 1 , which is also the time that the Greek government applied for assistance from the International Monetary Fund (IMF) and the European Union (EU). Together with the influence of the financial crisis and the European debt crisis, the curve falls fast until the lowest point, Point 2. Differently from the previous process, the bottoming out this time is rapid, even exceeding the range limit to Point 3. The key reason for the relatively large setback in this process is U.S. QE2. Compared with the other three instances of QE for America, the QE2 is special as it related only to the buy-back and sale of U.S. national debt, which is actually beneficial 
for the market and provides more energy for the rising process. After the peak at Point 3 , the European debt crisis continues fermenting, causing global stock market linkage to rise sharply again. The downward trend is stopped at the lowest point, Point 5.

The third stage can be discerned beginning from the $1,627^{\text {th }}$ observation, Point 5 , to the 2,547 th observation, Europe QE. Point 5 represents the event of the G20 finance ministers' meeting in Paris, as the European countries put forward a specific solution to the crisis, after which, with the U.S. QE3 and QE4, the American economic situation gradually improves. As the systemic risk is gradually weakened, the global stock markets slowly become sparse and show a steady recovery process on the curve. The U.S. withdraws from $\mathrm{QE}$ at the 2,420 th observation, causing the curve to pass through the trend line downward as the other markets' investments transfer to the markets of America affected by the policy, but then return to above the trend line.

From the Europe QE to the end is the fourth stage. The reason for saying that this is a possible trend is that the time series here is not sufficiently long to confirm the trend. Referring to the previous trend of the curve, the trend with a large probability is downward. In addition, it is possible that it will be an interval fluctuation.

To analysis the periodicity, we introduce a low-pass filter to smooth the curve for the reason mentioned in the research on volatility. First, we examine whether there is a seasonal trend in this curve and draw the conclusion that this not obvious. Then, we obtain the smooth curve indicated in Figure 6 by the short dashed line, with the original curve indicated by the solid line. Combined with the result of the tendency, we defined the cycle as a process from one lowest point to another, and because the time series included only the workdays, there are approximately 260 days in a year. The first stage is from the first to the $871^{\text {st }}$ observation. Especially, we regard the subperiod with the observations from the $451^{\text {st }}$ to $559^{\text {th }}$ and observations from the $559^{\text {th }}$ to $597^{\text {th }}$ as one

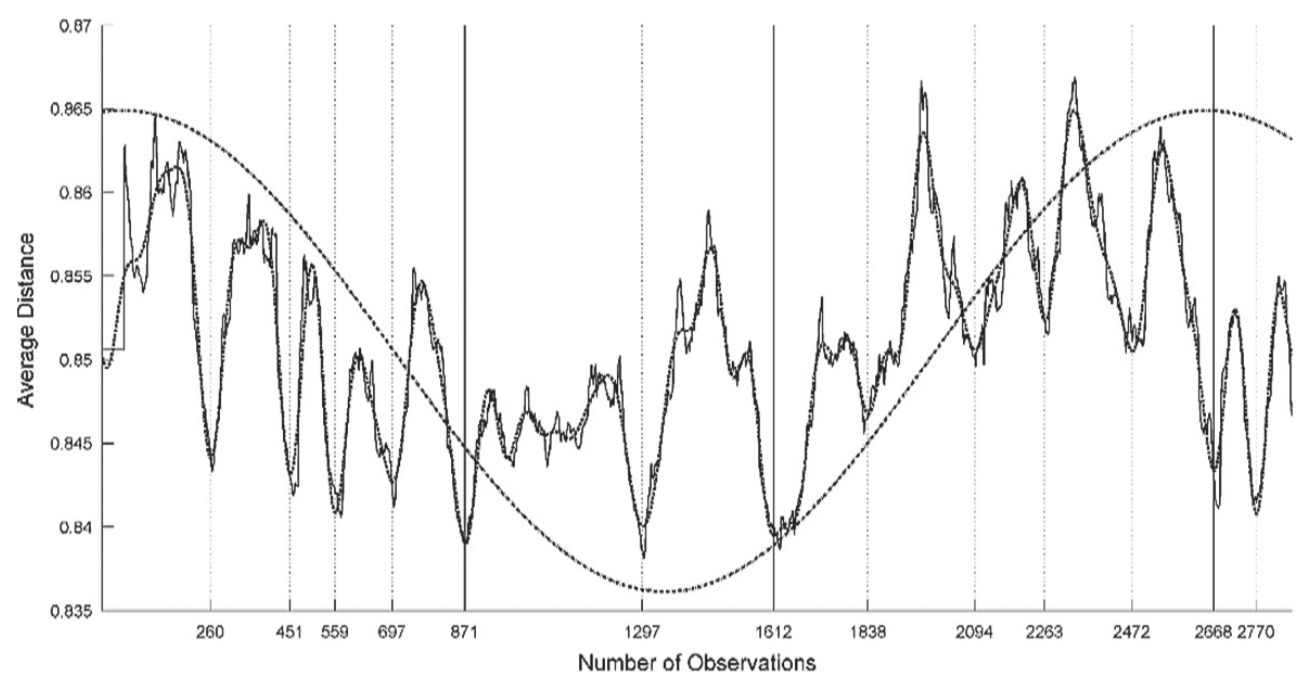

Fig. 6. Low frequency components of average distance 
subcycle, because the subprime mortgage crisis and the following measures induced in the markets an irrational fluctuation, and merging these two waves can deter reflection of the trend. Then, there are four subcycles covering 871 days, with the average fluctuation cycle being approximately 0.8375 years, hence we named it the pre-crisis cycle. Similarly, the second stage's cycle, the in-crisis cycle, is approximately 1.425 years, and the third stage's cycle, the recovery cycle, is approximately 0.8123 years. The cycle of stage four cannot be calculated as we lack sufficient data.

According to the result, the linkage cycle during the crisis is much longer than the cycle before and after the crisis, and the cycle before the crisis is also longer than the one after the crisis. The global stock market linkage cycle represents a "high-low-high" process. Before the financial crisis, the comovement is at a relatively high level, but during the period of the crisis, various stimulus policies, such as U.S. QEs, China's four trillion stimulus plan, EU rescue measures in Greece, are launched frequently, leading to different capital markets having individual differences. On the one hand, in order to withstand the impact of the financial crisis, cooperation in the region has been strengthened, which cause the regional stock market linkage increased. One the other hand, different degrees of trade protectionism and other policies make the global market divided, leading to a decline in inter-regional linkage. As a result, the comovement of the global stock market remained at a reduced level for a long time, which leads the global stock market linkage cycle to be longer. Frequent financial stimulus plans have made different countries to some extent affected by monetary policy from other countries. Then, the average distance between the global stock market declines, which means the linkage gradually increased as the world economy resuscitated slowly, largescale market intervention gradually reduced, and the linkage between regional markets restored. The difference between the cycle before and after the crisis, representing the linkage of the global stock market increasing, can be explained by the fact that global capital flows caused by the financial crisis leading to an overflow of the monetary policy from the developed countries to the developing countries. Besides, there are also reasons for global economic integration. Although the financial crisis has had a certain impact on the process of globalization, the trend of global financial integration is still obvious, and the recovery is faster after the crisis than the real economy.

In addition, we attempt to fit all of our series for the average distance using the trigonometric function and we obtain a fitting equation as

$$
y=0.0144 \cos \left(\frac{2 \pi}{2600}(x-50)\right)+0.8505 .
$$

The image of this equation is also shown in Figure 6 by a long dashed line. This result means that there exists a larger cycle for the global stock market linkage of approximately ten years and the trend of the average distances curve will be downward.

\subsection{Global stock market network importance measure}

The method of average weighted degree is used here to find the important indices, which is defined as the sum of the weighted degrees divided by the number of the non-zero correlation coefficients. As we have divided the time series into 13 parts using the set 
of subcycles, the average weighted degrees are also calculated using this partition. The top ten of the average weighted degrees' ranking in each period are shown in Table 2.

Comparing the ranking of the 13 stages, the index set is stable, and the change of the rank is small. As all of the indices are from Europe, the European stock markets have the biggest influence on global stock markets. Some similar conclusions also appear in previous studies (Gilmore et al. 2008). However, considering the cluster phenomenon, the results have occurred because the European stock markets comprise the biggest connected subgraph of the network. We reveal the hierarchical structure by analyzing the MST distance matrices using clustering. For every stage, we calculate the average distance of each node to all of the other points and can observe that stock markets in the same region have a higher linkage than stock markets in different regions. Then, ranking the average weighted degree for different areas is more meaningful than for all the areas. As there are only two indices from Africa, we calculate only the rank of the other three areas. For America, the main stock indices are NYA and SPX, indicating that the U.S. stock markets play a key role in America. FCHI, AEX, BFX and GDAXI are the important indices for European stock markets. Most of the Asia Pacific stock markets concern STI and HIS.

Table 2. Top ten of the average weighted degree's ranking in each period

\begin{tabular}{|c|c|c|c|c|c|c|c|c|c|c|}
\hline & 1 & 2 & 3 & 4 & 5 & 6 & 7 & 8 & 9 & 10 \\
\hline Stage1 & FCHI & AEX & BFX & UKX & DAXI & OMXSPI & ATX & ITLMS & HEX & IBEX \\
\hline Stage2 & FCHI & AEX & BFX & GDAXI & UKX & OMXSPI & ATX & HEX & ITLMS & IBEX \\
\hline Stage3 & FCHI & AEX & BFX & GDAXI & UKX & OMXSPI & ATX & ITLMS & HEX & IBEX \\
\hline Stag & FCHI & AEX & BFX & GD & UKX & OMXSPI & ATX & ITLMS & HEX & IBEX \\
\hline Stage 5 & FCHI & AEX & BFX & GDAXI & UKX & OMXSPI & ITLMS & ATX & HEX & IBEX \\
\hline Stage6 & FCHI & AEX & BFX & GDAXI & UKX & OMXSPI & ATX & ITLMS & HEX & IBEX \\
\hline Stage 7 & FCHI & AEX & BFX & GDAXI & UKX & 1XSPI & HEX & ATX & ITLMS & IBEX \\
\hline Stage 8 & FCHI & AEX & BFX & GDAXI & UKX & OMXSPI & ATX & HEX & ITLMS & IBEX \\
\hline Stage9 & FCHI & AEX & BFX & GDAXI & UKX & OMXSPI & ATX & HEX & ITLMS & IBEX \\
\hline ge 10 & AEX & FCHI & BFX & GDAXI & UKX & $\mathrm{OM}$ & ATX & HEX & ITLMS & IBEX \\
\hline Stage11 & FCHI & AEX & BFX & UKX & GDAXI & OMXSPI & ATX & HEX & ITLMS & IBEX \\
\hline Stage12 & FCHI & AEX & BFX & UKX & GDAXI & OMXSPI & HEX & ATX & ITLMS & IBEX \\
\hline Stage13 & FCHI & AEX & BFX & GDAXI & UKX & IXSPI & ATX & HEX & ITLMS & IBEX \\
\hline
\end{tabular}

\section{Conclusions}

From 2005 to 2016, global financial markets experienced significant volatility, including major economic crises such as the subprime mortgage crisis, the global financial crisis, the European debt crisis, as well as strong market intervention policies such as QEs. Financial integration has brought the global financial markets together, pushing the market to a higher level during the boom and increasing the market's destructive power in times 
of crisis. The global financial crisis of 2008 has greatly affected the development of the world economy. Today, the developed countries are still growing weak, and the growth rate of emerging market countries is slowing down. In the process of crisis recovery, the integration process has been questioned by its infective effects, some countries have begun to take trade protectionism, and even some European countries out of the EU.

For the global stock market, the direct impact of financial crisis and market intervention is reflected in the international flow of capital, the injection of capital market liquidity and the monetary policy spillover effect. This paper defined the impact of financial crises and bailout measures on global stock market linkage. The dynamic correlation coefficient of the volatility network topological characteristics, such as network density, average path length, clustering coefficient, can be considered as the parameters of the global stock market linkage to reflect the dynamic changes. At the same time, through the trend analysis of the relevant characteristics the influence on the global stock market linkage by the financial crisis and the major rescue measures can be confirmed. Our research shows that before the outbreak of the financial crisis, the global stock market linkage fluctuate violently, the linkage significantly improved when the crisis broke out, and QEs, as well as other rescue measures will make the global stock market linkage significantly reduced.

This paper also defined the variable periodicity of the global stock market linkage and carried on the empirical test. By smoothing the average distance curve calculated by the minimum spanning tree, the global stock market linkage cycle in different stages was identified as 0.8375 years before the financial crisis, 1.425 years during the crisis and 0.8123 years after the crisis. It implied that the after the financial crisis, the global stock market cycle is shorter than before the financial crisis. Further, we considered to study the global cycle of the world stock market linkage by using the trigonometric function curve and drew the conclusion that the global cycle of world stock index linkage is about 10 years.

In addition, the importance measure showed that even though the network properties are dynamic, the structure is relatively stable over time. As a result of the average weighted degree analysis, European stock markets play the key role in global stock markets, and the indices of NYA and SPX, of FCHI, AEX, BFX and GDAXI, and of STI and HIS are the important nodes of America, Europe and Asia Pacific, respectively. For the global stock markets, European stock markets are the information transmission hub, because the America and the Asia Pacific markets are connected by Europe. However, as the 2008 financial crisis prompted a prominent change in the global stock markets network, the U.S. is still the key to the global markets, because the U.S. is not the centre just because most of its information is spread by Europe.

Comparing with the previous researches which adopt a complex network framework to analyze the relationship between stock markets, this paper provided a globalization perspective to research the comovement of global stock markets instead of regional market. It would be a meaningful attempt as we have shown in the paper that different regional markets are mutually influenced, a biased conclusion may be led if we simply 
considered a regional market. There are also some scholars try to explain the synchronization in the global stock market by complex network. In their study, the networks were constructed according to the dynamic correlation coefficient derived from the rolling window model. Although the authors tried to use different window values to avoid the error caused by the rolling window, it is still not possible to avoid the impact of human factors on the results of the analysis. We provided the method of DCC-MVGARCH to calculate the dynamic correlation coefficient, not only avoids the interference of human factors, but also solves the problem of Heteroscedasticity in time series. Besides, instead of dividing the global stock market into developed markets, emerging markets and frontier markets, we believed it would be more appropriately to partition the market as European stock market, America stock market, Asia Pacific stock market and MENA stock market as the global stock markets show obvious regional aggregation.

Another important contribution of this article is that we attempted to study global stock market comovements from the perspective of not only the financial events regarding the crisis but also such stimulus measure as QEs and other important international meetings. In contrast to the classification method that just regards the 2008 financial crisis as the break point or the 2008 financial crisis and the European debt crisis as the break points for dividing the time series into two or three parts, we could divide the dynamic curve into four parts in a more reasonable manner. In addition, we attempted to investigate the dynamic interaction cycle in terms of networks and image processing instead of traditional econometrics, which is also a meaningful attempt.

By the methods of MST and low pass filter, we provided a new method to calculate the variable cycle of the global stock market linkage and got the attractive phenomenon that the linkage cycle after the crisis became shorter than the linkage cycle before the crisis which means that this approach may offer a new perspective for studying the relationship between the stock markets linkage and economic cycles. Though the economic cycle has been extensively explored using econometric methods, we extended cycle research from being data driven to having data combined with financial events. Considering the financial events in conducting the analyses of whether there are important connections between the linkage and economic cycles is meaningful research.

Although analyzing the comovement of the global stock market by a globalization perspective through DCC-MVGARCH to obtain a dynamic MST and low pass filter is meaningful, there exists some limitations on the data set and the volatility network. First of all, using daily closing data from regions in different time zones implies that the returns are asynchronous and some markets close systematically earlier than other, which allows more information to be incorporated into the returns. The existing literature on this subject mainly includes three categories: using daily return data, using weekly return data and set a lag period. Whether it is the daily return or weekly rate of return, strictly speaking, errors always exist. A meaningful attempt may be to determine the lag relationship between different pair of markets. Further, the global stock market linkage should actually be represented as a directed graph, and the interaction between the same market pairs should be different, how to determine the influence of each other is also a meaningful subject. 
The conclusions are enlightening. On the one hand, from the perspective of application, with the trend and cycle of the linkage, the government can better grasp the trend of the global stock markets and then pay more attention to the key indices rather than to the entire system to strengthen the forecast and control of possible risk. On the other hand, it is also significant for policymakers to confirm the clustering structure that the local stock market is in, clear the path of information transmission by the network and then formulate a targeted stock market development program.

\section{Acknowledgment}

The author are very grateful for the insightful comments and suggestions of the anonymous reviewers. Furthermore, the relevant researches done in this paper are supported by The national social science fund major projects (14ZDB151); Public welfare industry research projects $(201305034,201405029)$; The ministry of education philosophy and social sciences development report breeding project (13JBGP005); General Financial Grant from the China Postdoctoral Science Foundation (2015M580611); Qingdao postdoctoral application research project funding (251); Fundamental Research Funds for the Central Universities (201613006, 201564031), the National Natural Science Foundation of China (Nos. 71471172 and 71271124), the Special Funds of Taishan Scholars Project of Shandong Province (No. ts201511045).

\section{Reference}

Aslanidis, N.; Osborn, D.; Sensier, M. 2010. Co-movements between US and UK stock prices: the role of time-varying conditional correlations, International Journal of Finance \& Economics 15(4): 366-380.

Aste, T.; Shaw, W.; Di Matteo, T. 2010. Correlation structure and dynamics in volatile markets, New Journal of Physics 12(8): 085009. https://doi.org/10.1088/1367-2630/12/8/085009

Bessler, D. A.; Yang, J. 2003. The structure of interdependence in international stock markets, Journal of International Money and Finance 22(2): 261-287.

https://doi.org/10.1016/S0261-5606(02)00076-1

Boginski, V.; Butenko, S; Pardalos, P. M. 2005. Statistical analysis of financial networks, Computational Statistics \& Data Analysis 48(2): 431-443.

https://doi.org/10.1016/j.csda.2004.02.004

Bollerslev, T. 1990. Modeling the coherence in short-run nominal exchange-rates - a Multivariate Generalized Arch Model, Review of Economics and Statistics 72(3): 498-505. https://doi.org/10.2307/2109358

Brida, J. G.; Risso, W. A. 2010. Hierarchical structure of the German stock market, Expert Systems with Applications 37(5): 3846-3852. https://doi.org/10.1016/j.eswa.2009.11.034

Brida, J. G.; Matesanz, D.; Seijas, M. N. 2016. Network analysis of returns and volume trading in stock markets: the euro stoxx case, Physica: Statistical Mechanics and its Applications 444: 751-764.

Buttfield, A.; Bolton, M. 2005. Real time measurement of RR intervals using a digital signal processor, Journal of Medical Engineering \& Technology 29(1): 8-13.

https://doi.org/10.1080/0309190041000166234

Caraiani, P. 2013. Using complex networks to characterize international business cycles, Plos One 8(3): e581093. https://doi.org/10.1371/journal.pone.0058109 
Carley, K. M.; Malik, M.; Landwehr, P. M.; Pfeffer, J.; Kowalchuck, M. 2016. Crowd sourcing disaster management: the complex nature of twitter usage in padang Indonesia, Safety Science 90: 48-61. https://doi.org/10.1016/j.ssci.2016.04.002

Chan, L. 2006. How does the Asian crisis affect the interdependencies between major financial markets in Asia and the US, Journal of Emerging Markets 10(2): 25-34.

Chandra, M. 2005. Estimating and explaining extreme comovement in Asia-Pacific equity markets, Review of Pacific Basin Financial Market and Policies 8(2): 53-79. https://doi.org/10.1142/S0219091505000348

Cheong, S. A.; Fornia, R. P.; Lee, G. H. T.; Kok, J. L.; Yim, W. S.; Xu, D. Y.; Zhang, Y. 2012. The Japanese economy in crises: a time series segmentation study, Economics: the Open Access, Open-Assessment E-Journal 6(5): 1-81. https://doi.org/10.5018/economics-ejournal.ja.2012-5

Chi, K.; Jing, L.; Lau, F.; Baillie, R.; Palm, F. 2010. A network perspective of stock market, Journal of Empirical Finance 17(4): 659-667. https://doi.org/10.1016/j.jempfin.2010.04.008

Cho, D. C.; Eun, C. S.; Senbet, L. W. 1986. International arbitrage pricing theory: an empirical investigation, Journal of Finance 41(2): 313-329.

https://doi.org/10.1111/j.1540-6261.1986.tb05038.x

Coletti, P. 2016. Comparing minimum spanning trees of the Italian stock market using returns and volumes, Physica a: Statistical Mechanics and its Applications 463: 246-261.

Connolly, R. A.; Wang, F. A. 2003. International equity market comovements: economic fundamentals or contagion?, Pacific-Basin Finance Journal 11(1): 23-43.

https://doi.org/10.1016/S0927-538X(02)00060-4

Contessi, S.; Pace, P.; Francis, J. 2008. The cyclical properties of disaggregated capital flows, Journal of International Money \& Finance 32: 528-555.

https://doi.org/10.1016/j.jimonfin.2012.05.023

Égert, B.; Kočenda, E. 2007. Interdependence between eastern and western European stock markets: evidence from intraday data, Economic Systems 31(2): 184-203.

https://doi.org/10.1016/j.ecosys.2006.12.004

Engle, R.; Kroner, K. 1995. Multivariate simultaneous generalized ARCH, Econometric Theory 11(1): 122-150. https://doi.org/10.1017/S0266466600009063

Engle, R. 2002. Dynamic conditional correlation: a simple class of multivariate generalized autoregressive conditional heteroskedasticity models, Journal of Business \& Economic Statistics 20(3): 339-350. https://doi.org/10.1198/073500102288618487

Eryigit, M.; Eryigit, R. 2009. Network structure of cross-correlations among the world market indices, Physica A: Statistical Mechanics and its Applications 388(17): 3551-3562.

https://doi.org/10.1016/j.physa.2009.04.028

Eun, C. S; Shim, S. 1989. Intemational transmission of stock market movement, The Journal of Financial and Quantitative Analysis 24(2): 241-256. https://doi.org/10.2307/2330774

Fan, R. G. 2014. Collaborative innovation in social governance in a complex network structural paradigm, Social Sciences in China 4: 98-120.

Fang, J.; Wang, X.; Zheng, Z.; Bi, Q.; Di, Z.; Li, X. 2007. New interdisciplinary science: network science, Progress in Physics 27: 239-343.

Gao, X.; An, H.; Zhong, W. 2013. Features of the correlation structure of price indices, Plos One 8(4): e610914. https://doi.org/10.1371/journal.pone.0061091

Garas, A.; Argyrakis, P. 2007. Correlation study of the Athens stock exchange, Physica A: Statistical Mechanics and its Applications 380: 399-410. https://doi.org/10.1016/j.physa.2007.02.097

Gilmore, C. G.; Lucey, B. M.; Boscia, M. 2008. An ever-closer union? Examining the evolution of linkages of European equity markets via minimum spanning trees, Physica A: Statistical Mechanics and its Applications 387(25): 6319-6329. https://doi.org/10.1016/j.physa.2008.07.012 
Gong, P.; Huang, R. 2009. Empirical analysis of sub-prime mortagage crisis's impacts on Chinese stock market: based on the interaction between Chinese and American stock market, Management Review 2: 21-32.

Guyot, A; Lagoarde-Segot, T.; Neaime S. 2014. Foreign shocks and international cost of equity destabilization: evidence from the MENA region, Emerging Markets Review 18: 101-122. https://doi.org/10.1016/j.ememar.2014.01.003

Huang, S.; Chen, F.; Chen, L. 2017. Global dynamics of a network-based siqrs epidemic model with demographics and caccination, Communications in Nonlinear Science and Numerical Simulation 43: 296-310. https://doi.org/10.1016/j.cnsns.2016.07.014

Huang, W.; Zhuang, X.; Yao, S. 2009. A network analysis of the Chinese stock market, Physica A: Statistical Mechanics and its Applications 388(14): 2956-2964.

https://doi.org/10.1016/j.physa.2009.03.028

Huang, W. Q.; Zhuang, X. T.; Yao, S.; Urysaev, S. 2016. A financial network perspective of financial institutions' systemic risk contributions, Physica A: Statistical Mechanics and its Applications 456: 183-196.

Hwang, S.; Kim, Y. I.; Shin, J. 2016. An analysis of herding in the Korean stock market using Network Theory. Korea: Social Science Electronic Publishing.

Jiang, B. 2016. A complex-network perspective on Alexander's wholeness, Physica A: Statistical Mechanics and its Applications 463: 475-484. https://doi.org/10.1016/j.physa.2016.07.038

Jung, W. S.; Chae, S.; Yang, J. S.; Moon, H. T. 2006. Characteristics of the Korean stock market correlations, Physica A: Statistical Mechanics and its Applications 361(1): 263-271.

https://doi.org/10.1016/j.physa.2005.06.081

Karolyi, G.; Stulz, R. 1996. Why do markets move together? An investigation of U.S.-Japan stock return comovements, Journal of Finance 51(3): 951-986.

https://doi.org/10.1111/j.1540-6261.1996.tb02713.x

Lee, J.; Om, K.; Choi, M.; Song, C; Kim, K. 2014. Scientists and engineers in convergence technologies in Korea: where are they going and how do they collaborate?, Technological and Economic Development of Economy 20(3): 434-456. https://doi.org/10.3846/20294913.2014.880388

Liang, Q.; Li, Z.; Hao, X. 2015. The internationaization of Chinese stock market: based on information spillover, Economic Research Journal 50(4): 150-164.

Liu, X. F.; Tse, C. K. 2012. A complex network perspective of world stock markets: synchronization and volatility, International Journal of Bifurcation and Chaos 22(6): 1250142.

https://doi.org/10.1142/S0218127412501428

Lyócsa, Š.; Výrost, T.; Baumöhl, E. 2012. Stock market networks: the dynamic conditional correlation approach, Physica A: Statistical Mechanics and its Applications 391: 4147-4158.

https://doi.org/10.1016/j.physa.2012.03.038

Ma, P.; Li, D.; Li, S. 2016. Efficiency and cross-correlation in equity market during global financial crisis: evidence from China, Physica A: Statistical Mechanics and its Applications 444: 163-176. https://doi.org/10.1016/j.physa.2015.10.019

Majapa, M.; Gossel, S. J. 2016. Topology of the South African stock market network across the 2008 financial crisis, Physica A: Statistical Mechanics and its Applications 445: 35-47.

https://doi.org/10.1016/j.physa.2015.10.108

Mantegna, R. N. 1999. Information and hierarchical structure in financial markers, Computer Physics Communications 121: 153-156. https://doi.org/10.1016/S0010-4655(99)00302-1

Mylonidis, N.; Kollias, C. 2010. Dynamic European stock market convergence: evidence from rolling cointegration analysis in the first euro-decade, Journal of Banking \& Finance 34(9): 2056-2064. https://doi.org/10.1016/j.jbankfin.2010.01.012

Naylor, M. J.; Rose, L. C.; Moyle, B. J. 2007. Topology of foreign exchange markets using hierarchical structure methods, Physica A: Statistical Mechanics and its Applications 382(1): 199-208. https://doi.org/10.1016/j.physa.2007.02.019 
Neaime, S. 2006. Volatilities in emerging MENA stock markets, Thunderbird International Business Review 48(4): 455-484. https://doi.org/10.1002/tie.20105

Neaime, S. 2012. The global financial crisis, financial linkages and correlations in returns and volatilities in emerging MENA stock markets, Emerging Markets Review 13(2): 268-282.

https://doi.org/10.1016/j.ememar.2012.01.006

Neaime, S. 2016. Financial crises and contagion vulnerability of MENA stock markets, Emerging Markets Review 27: 14-35. https://doi.org/10.1016/j.ememar.2016.03.002

Neely, C.; Weller, P. 2000. Predictability in international asset returns: a reexamination, Journal of Financial \& Quantitative Analysis 35(4): 601-620. https://doi.org/10.2307/2676257

Nobi, A.; Lee, S.; Kim, D. H.; Lee, J. W. 2014. Correlation and network topologies in global and local stock indices, Physics Letters A 378(34): 2482-2489.

https://doi.org/10.1016/j.physleta.2014.07.009

Park, D.; Kim, G.; On, B. 2016. Understanding the network fundamentals of news sources associated with a specific topic, Information Sciences 372: 32-52.

https://doi.org/10.1016/j.ins.2016.08.015

Porat, I.; Benguigui, L. 2016. Global migration topology analysis and modeling of bilateral flow network 2006-2010, EPL (Europhysics Letters) 115(1): 18002.

https://doi.org/10.1209/0295-5075/115/18002

Qiao, H.; Xia, Y.; Li, Y. 2016. Can network linkage effects determine return? Evidence from Chinese stock market, Plos One 11(6): e01567846. https://doi.org/10.1371/journal.pone.0156784

Sensoy, A.; Tabak, B. M. 2014. Dynamic spanning trees in stock market networks: the case of Asia-Pacific, Physica A: Statistical Mechanics and its Applications 414: 387-402.

https://doi.org/10.1016/j.physa.2014.07.067

Shan, W.; Liu, C.; Yu, J. 2014. Features of the discipline knowledge network: evidence from China, Technological and Economic Development of Economy 20(1): 45-64.

https://doi.org/10.3846/20294913.2014.825460

Sui, Y.; Shao, F.; Wang, C.; Sun, R.; Ji, J. 2016. Complex network modeling of spectral remotely sensed imagery: a case study of massive green algae blooms detection based on modis data, Physica A: Statistical Mechanics and its Applications 464: 138-148.

https://doi.org/10.1016/j.physa.2016.07.061

Tabak, B. M.; Serra, T. R.; Cajueiro, D. O. 2010. Topological properties of stock market networks: the case of Brazil, Physica A: Statistical Mechanics and its Applications 389(16): 3240-3249.

Tsay, R. 2014. Multivariate time series analysis: with $R$ and financial applications. 1st ed. Wiley, 428-433.

Tumminello, M.; Aste, T.; Di Matteo, T.; Mantegna, R. N. 2005. A tool for filtering information in complex systems, Proceedings of the National Academy of Sciences of the United States of America 102(30): 10421-10426. https://doi.org/10.1073/pnas.0500298102

Tumminello, M.; Coronnello, C.; Lillo, F.; Micciche, S.; Mantegna, R. N. 2007. Spanning trees and bootstrap reliability estimation in correlation-based networks, International Journal of Bifurcation and Chaos 17(7): 2319-2329. https://doi.org/10.1142/S0218127407018415

Wang, G.; Xie, C. 2015. Correlation structure and dynamics of international real estate securities markets: a network perspective, Physica A: Statistical Mechanics and its Applications 424: 176-193. https://doi.org/10.1016/j.physa.2015.01.025

Wang, R.; Li, J.; Wang, L.; Yang, Y.; Lin, P.; Wu, Y. 2016. Application of complex network method to Ssatiotemporal patterns in a neuronal network, Physica A: Statistical Mechanics and its Applications 463: 219-230. https://doi.org/10.1016/j.physa.2016.07.046

$\mathrm{Xu}$, Y.; Wang, X.; Jia, J. 2010. An analysis of the linkage between Chinese and international stock markets: a research based On DCC-GARCH model, Economic Survey (05): 124-128. 
Kedong YIN, received the B.S. degree in system engineering from Nanjing University of Science and Technology, Nanjing, China, in 1988, M.S. degree in economics from Ocean University of China, Qingdao, China, in 1997, and $\mathrm{PhD}$ degree in business administration from Huazhong University of Science and Technology, Wuhan, China, in 2009. He is currently a Professor with the School of Economics, Ocean University of China, Shandong, China. He has authored or coauthored more than 100 publications. His research interests focus on quantitative economic analysis and modeling, financial risk management, and marine econometrics.

Zhe LIU, received the B.S. degree in mathematical economics from Shandong University. He is currently a graduate student at the School of Economics, Ocean University of China, Shandong, China. His research interests includes financial risk management, data mining and artificial intelligence.

Peide LIU, received the B.S. and M.S. degrees in signal and information processing from Southeast University, Nanjing, China, in 1988 and 1991, respectively, and the PhD degree in information management from Beijng Jiaotong University, Beijing, China, in 2010. He is currently a Professor with the School of Management Science and Engineering, Shandong University of Finance and Economics, Shandong, China. He is an Associate Editor of the Journal of Intelligent and Fuzzy Systems, the member of editorial board of the journal Technological and Economic Development of Economy, and the members of editorial board of the other 12 journals. He has authored or coauthored more than 150 publications. His research interests include aggregation operators, fuzzy logic, fuzzy decision making, and their applications. 